$\S=-1$

\title{
Hybrid modulation schemes for data transmission improvement of indoor visible light communication system
}

\author{
Abdelhalim Zekry ${ }^{1}$, Christena Ghandour ${ }^{2}$, Nazmi A. Mohammed ${ }^{3}$, S. El-Rabaie ${ }^{4}$ \\ ${ }^{1}$ Faculty of Engineering, Ain Shams University, Cairo, Egypt \\ ${ }^{2}$ Faculty of Engineering, Ain Shams University, Cairo, Egypt \\ ${ }^{3}$ New Cairo Academy for Science and Arts, New Cairo, Egypt \\ ${ }^{4}$ Faculty of Electronic Engineering, Menouf, Monoufia University, Menoufia, Egypt \\ *Corresponding author E-mail: christ.ghandour@yahoo.com
}

\begin{abstract}
This work enhances the bit rate characteristics, receiver sensitivity and power requirements of multicarrier modulation schemes (MCM) for visible light communication (VLC) dimming control system at bit error rate (BER) less than $10^{-3}$. This study develops the mathematical formulation for merging pulse position modulation (PPM) and overlapping pulse position modulation (OPPM) with M-ary quadrature amplitude modulation DC-Biased optical orthogonal frequency division multiplexing (M-QAM DCO OFDM), which can achieve efficient data transmission while maintaining communication quality. These schemes are then compared with the conventional merging (i.e M-QAM DCO OFDM with pulse width modulation (PWM)). Relating to the recent advances in the field, the additional comparative study is established with the latest merging platform (i.e. M-QAM DCO OFDM with multiple pulse position modulation (MPPM)).
\end{abstract}

Keywords: Visible Light Communication (VLC); M-ary Quadrature Amplitude Modulation DC-Biased Optical Orthogonal Frequency Division Multiplexing (M-QAM DCO OFDM); Pulse Width Modulation (PWM); Pulse Position Modulation (PPM); Overlapping Pulse Position Modulation (OPPM); and Multiple Pulse Position Modulation (MPPM).

\section{Introduction}

The need for alternative communication technologies is a result of increased wireless data traffic from the quickly growing wireless mobile devices, which is inspiring compression on the dwindling radio frequency (RF) spectrum. This interest about spectrum led to the formularization of a new technology called visible light communication (VLC) [1-3].

Additional security, the obscurity of interference with neighbor technology, free/large license bandwidth and availability of existing infrastructure are the main attracting features in VLC technology today [4-6].

One of the major traits in VLC based applications is that LED is the primary design element. High efficiency, low production cost, low power consumption, availability to operate at high bit rates and easy to integrate with existing lighting infrastructure are some of the merits of LEDs [3], [7-10]. In addition, LED benefits effectively from simple intensity modulation (IM) and direct detection (DD) to establish VLC based links [11] where the LED type used in this work is power LED.

Several interesting applications are achieved using VLC in recent years: Localization [12- 13], video streaming [14], traffic control systems [15], underwater transmission systems [16], biomedical [17], and effective data broadcasting within homes and offices [18]. High-efficiency VLC system, which should ideally merge high data rate, simple design, low power consumption, efficient dimming/flicker control and low as possible BER, are the target performance parameters of VLC communications [1, 7].

It is noteworthy to mention that achieving perfect performance regarding all the aforementioned characteristics is a difficult task.
A trade-off between the previously mentioned VLC characteristics is discussed in several kinds of literature [1-2]. Several Modulation schemes and architectures are proposed to provide efficient VLC systems with good/ acceptable performance regarding previous characteristics and taking into consideration the trade-off between different techniques [1-2], [19]. Modulation schemes can be classified basically as single carrier modulation (SCM) schemes and multicarrier modulation (MCM) [19].

SCM techniques are straightforward to implement in Li-Fi. OnOff Keying (OOK), pulse position modulation (PPM), overlapping pulse position modulation (OPPM), and M-ary pulse amplitude modulation (M-PAM) are techniques of modulation which can be easily implemented. These techniques are used in low to moderate data rates applications. When increasing the data rate, the BER of SCM techniques deteriorates because the inter-symbol interference (ISI) increases. So, such schemes require complex equalizers at the receiver to prevent any possible flickering due to the dispersive nature of optical wireless channels. Therefore, the performance (i.e BER) of SCM schemes degrades as their spectral efficiency (SE) increases [19].

MCM techniques offer high data rates solution that can adapt the system performance to the channel frequency response, support adaptive power, spectral and computational efficiency. This can maximize the system performance (BER) and minimize the complexity [19].

The signal of pulse width modulation (PWM) dimming control systems is only transmitted during the "on" period of PWM pulses. For OOK signals, the required data rate is inversely proportional to the duty cycle to keep data rate constant which makes the system hard to be implemented on the circuit level and causes more power consumption when the duty cycle is small. The com- 
bining of PWM dimming method with adaptive M-ary quadrature amplitude modulation DC-biased optical orthogonal frequency division multiplexing (M-QAM DCO OFDM) is proposed to solve several previous mentioned problems. However, in order to keep communication quality, the reduction of duty cycle in dimming will still cause increased symbol rate and consequently LED power. This means that the bit error rate (BER) performance gets degradation and the power consumption of the VLC system decreases [20-21].

In order to compensate for the decreased data rate when dimming, a method to combine variable M-QAM DCO OFDM signal with multiple pulse position modulation (MPPM) dimming control pulses is proposed in [22]. By using MPPM pattern encoding, excess data transmission can be achieved and the reduced data rate due to dimming control can be compensated. It is verified that proposed method can allow reduction of QAM symbol rate and required receiver sensitivity while stretching the range of reliable constant rate transmission [22-24].

This work introduces an elaborated modeling and simulation of the compensating effect of the different pulse position modulation techniques on the conventional M-QAM DCO OFDM PWM modulation dimming techniques. This is done by replacing PWM by MPPM pulses, or PPM or OPPM pulses with the same duty cycle, then assessing the performance of these techniques. The main performance parameter is the bit error rate for energy per bit to noise density ratio. Then for a given bit rate and bit error rate one, the achievable bit rate, required receiver sensitivity and required LED power are determined.

The rest of this paper is organized as follows: In Sec. 2, the mathematical model of the system is derived. The system model and modulation schemes structures are presented in subsection 2. 1, subsection 2.2 presents the merging schemes model and evaluating parameters. The analyzed performance is included in Sec. 3. Finally, Sec. 4 gives the conclusion.

\section{Mathematical model}

\subsection{System model and modulation schemes structures}
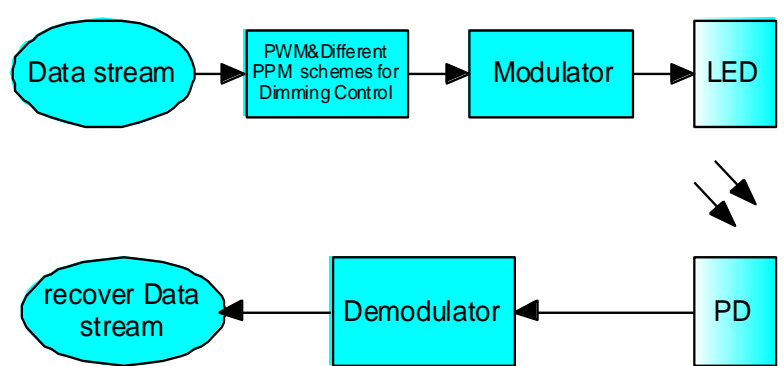

Fig. 1: The General Block Diagram of VLC System with Dimming Control.

Presuming that the transmitter as shown in Fig. 1 utilizes a general Lambertian radiation pattern, the channel direct current gain (i.e channel transfer function) can be presented as [3]

$$
\cdot \mathrm{H}(0)=\frac{(\mathrm{M}+1) A \cos ^{\mathrm{M}}(\Phi) \cos (\Psi)}{2 \pi d^{2}} .
$$

Where the Lambert coefficient of the sender is $\mathrm{M}=\frac{\ln (1 / 2)}{\ln (\cos (\Phi 1 / 2)}$

[20], the physical area of a detector is $A$, the transmission distance is $d$, the angle of irradiance is $\Phi$ and the angle of incidence is $\Psi$. Where $0 \leq \Psi \leq \Psi_{c}$, the field of view (FOV) of a receiver is $\Psi_{c}$. The received carrier power can be written as $\mathrm{P}_{r}={ }^{\cdot} \mathrm{H}(0) \mathrm{P}_{t}$ where the LED lamp power without modulation is $\mathrm{P}_{t}$ [25]. The signalto-noise ratio (SNR) of the output electrical signal can be presented after photo-detection as [2]
$S N R=\frac{\left(\Re \mathrm{P}_{r} M_{\text {index }}\right)^{2} f(\mathrm{t})^{2}}{\sigma^{2}}$

Where $\mathfrak{R}$ is the responsivity of the photo-detector (The photodetector consists of a photo diode followed by field effect transistor amplifier (FET)), $M_{\text {index }}$ represents the modulation depth and $f(\mathrm{t})$ gives the normalized modulating signal, respectively. $f(t)=|y| / \sqrt{p_{\text {avg }}}$ where $y$ is the transmitted signal that has different forms corresponding to the modulation schemes. For pulse position modulation (PPM), $y_{P P M}$ can be expressed by $y_{P P M}=L_{P P M} P \sum_{k=0}^{L_{P P M}} c_{k} p\left(t-\frac{k T}{L_{P P M}}\right)$. For multiple pulse position modulation (MPPM), $y_{M P P M}$ can be put in the form $y_{M P P M}=\frac{w \sqrt{n T}}{p} \sum_{k=0}^{L_{M P M M}} c_{k} \Phi\left(t-\frac{k T}{L_{M P P M}}\right) \cdot$ For overlapping pulse position modulation (OPPM), $y_{O P P M}$ can be formulated as: $y_{\text {OPPM }}=\sum_{k} P_{\text {peak }} p_{T_{D}}^{l(\mathrm{k})}\left(t-\frac{k T}{L_{O P P M}}\right)$. Finally, the orthogonal frequency division multiplexing (OFDM) $y_{O F D M}$ is given by $\left.y_{\text {OFDM }}=\frac{2}{\sqrt{2 N}} \sum_{k=0}^{N-1}\left|Y_{k}\right| \cos \left(2 \Pi \mathrm{k} \frac{n}{2 N}+\arg \left(Y_{k}\right)\right)\right)$ where $T$ is the duration of the symbol interval, $\mathrm{p}(\mathrm{t})$ is a rectangular pulse of unit subintervals, $\left[\mathrm{c}_{0}, \mathrm{c}_{1}, \mathrm{c}_{2}, \ldots, \mathrm{c}_{\mathrm{L}-1}\right]$ are the code words, $\Phi(t)$ is a rectangular pulse of unit energy, $w$ is the number of slots of an MPPM, $\mathrm{p}$ is the number of pulses in MPPM $p_{T_{D}}^{l(\mathrm{k})}(\mathrm{t})=1$ for $1(\mathrm{k}) \frac{T}{L_{O P P M}} \leq t \leq[1(\mathrm{k})+w] \frac{T}{L_{O P P M}} \quad, \quad p_{T_{D}}^{l(\mathrm{k})}(\mathrm{t})=0$ elsewhere, $p_{\text {peak }}$ is peak optical power, $2 N$ is the number of subcarrier, $Y_{k}=Y_{2 N-k}^{*}$ is the conjugate symmetry constraint and $p_{a v g}$ is the average optical power. Here, it is assumed that the main classifications of noise variance $\sigma^{2}$ are shot noise and thermal noise, which can be presented by [3], [20], [23]

$$
\begin{aligned}
& \sigma^{2}=\sigma^{2}(\mathrm{~B}, \mathrm{R})=\sigma_{\text {shot }}^{2}+\sigma_{\text {thermal }}^{2} \\
& \sigma_{\text {shot }}^{2}(B, R)=2 q\left[\mathfrak{R \mathrm { P } _ { r }}\left(1+\left(M_{\text {index }} f(t)\right)^{2}\right)+I_{b g} I_{2}\right] B \\
& \sigma_{\text {thermal }}^{2}(B)=\frac{8 \pi k \mathrm{~T}_{k} \mu A B^{2} I_{2}}{G}+\frac{16 \pi^{2} k \mathrm{~T}_{k} \Gamma B^{3} \mu^{2} A^{2} I_{3}}{g_{m}}
\end{aligned}
$$

Where the electronic charge is $q$, equivalent noise bandwidth is $\mathrm{B}$, background current is $\mathrm{I}_{\mathrm{bg}}$, the noise bandwidth factors is $\mathrm{I}_{2}$, the two terms demonstrate feedback resistor noise, and $\Gamma$ is FET channel noise respectively. Here, Boltzmann's constant is $k$, absolute temperature is $T_{k}$, the open-loop voltage gain is $\mathrm{G}, \mu$ is the fixed capacitance of photo-detector per unit area and $g_{m}$ is the FET transconductance. The all previous parameters are presented in [3], [22], [24].

Signals are only transmitted within the "on" period of PWM pulses in conventional PWM dimming control systems. Thus, for intensity modulation signals, if it is required to keep the average data rate constant, the M-QAM required data rate after dimming is inversely-proportional to duty cycle $D$ [22-24] which can be represented as 


$$
R_{1}=\frac{R_{0}}{D} \quad(0<D \leq 1)
$$

Where the initial data rate at the duty cycle is represented by $R_{0}$ and rate after dimming is $R_{1}$.

In this work, schemes combining (PPM, OPPM, and MPPM) light pulses and M-QAM OFDM signal with each other are proposed. Traditional PWM dimming pulses are divided into PPM or OPPM or MPPM pulses with the same duty cycle. Before presenting the proposed merged model in subsection 2.2, a general system schematic is presented in Fig.1 and Fig. 2. This schematic is then followed by a review of the patterns and structures of PWM, PPM, OPPM, and MPPM pulses before and after merging as shown in Fig. 3. In the same part, this work presents highlights about converting the dimming mechanism of PWM to PPM and OPPM based on the conversion that is done before (i. e PWM to MPPM [20], [22]).

Figure 2 shows the system schematic with applying the proposed dimming schemes. Data stream passes through PWM, PPM, OPPM or MPPM blocks to dim the brightness of LEDs. Then the signal passes to M-QAM DCO OFDM modulation. The modulated signal is then applied to the LED to complete the transmitter path. The receiver performs the inverse processing on the detected signal where the signal after transmission is received by the photodetector (PD). The received signal is then decomposed to its main components: The M-QAM DCO OFDM and one of the dimming schemes PWM, PPM, OPPM or MPPM where it is demodulated. At the end, the sent information bits are recovered.

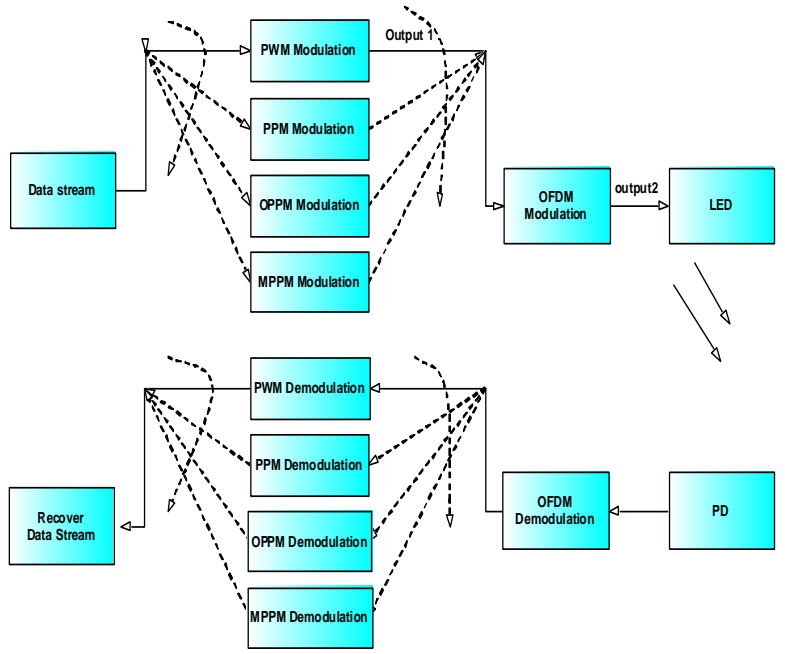

Fig. 2: System Block Diagram for Combining (PWM, PPM, OPPM or MPPM) Patterns with M-QAM DCO OFDM Signals.

To demonstrate the signal processing in the VLC transmitter, we displayed signal waveforms at the output of the dimmer at point 1 and point 3 in Fig. 2 at the output of the OFDM block. The patterns of conventional PWM, PPM, OPPM or MPPM pulses, which are at the output point 1 of Fig. 2 and after being merging with 4 QAM DCO OFDM at the output 2 in Fig. 2, are shown in Fig. 3.
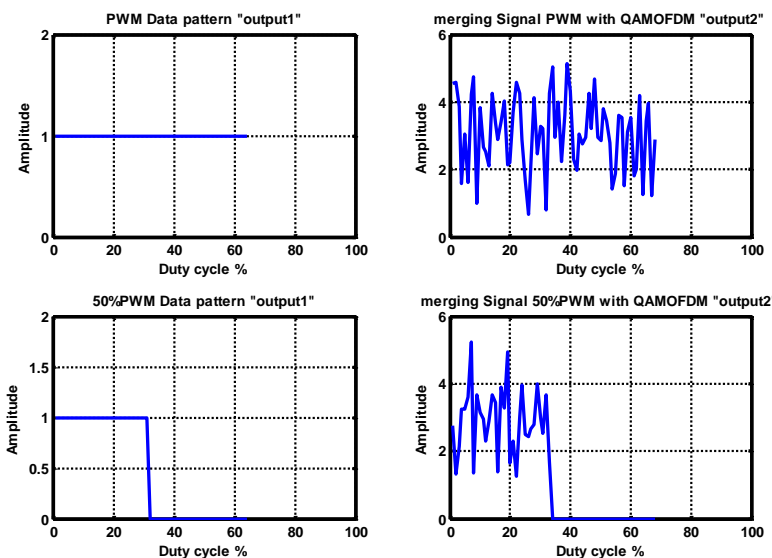

Fig. 3: A) PWM Structure and Bipolar OFDM Signal Waveforms Transmitted at the "on" Period of PWM Pulses. Duty Ratio 100\% and 50\%.
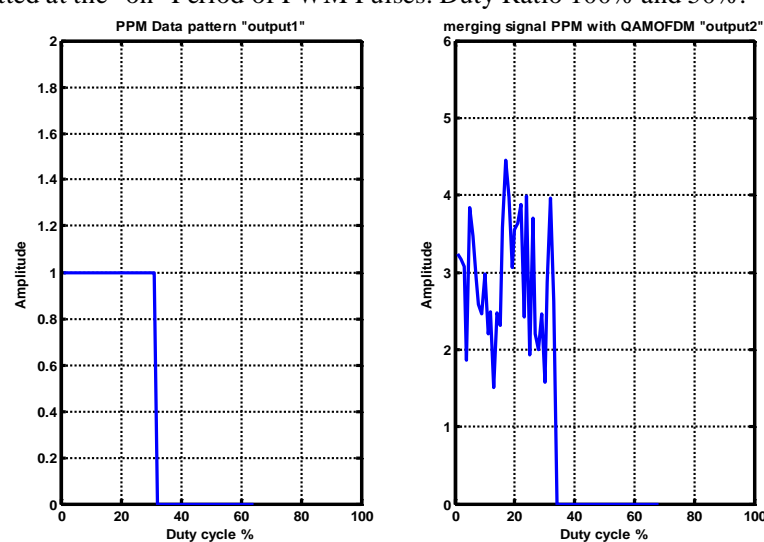

Fig. 3: B) Dividing A PWM Pulse with 50\% Dimming Into A PPM and Bipolar OFDM Signal Transmitted at the "on" Period of PPM Pulses.
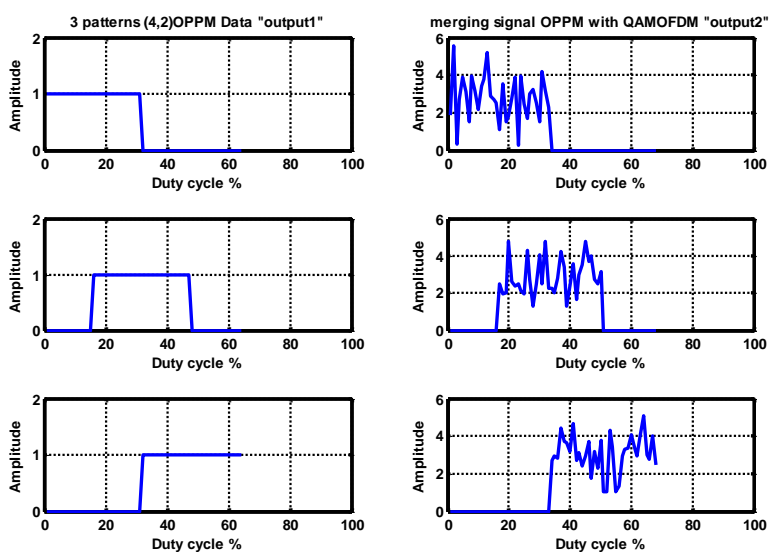

Fig. 3: C) Dividing A PWM Pulse with 50\% Dimming Into A (W=4, P=2) OPPM Symbol and Bipolar OFDM Signal Transmitted at the "on" Period of $(4,2)$ OPPM Patterns.
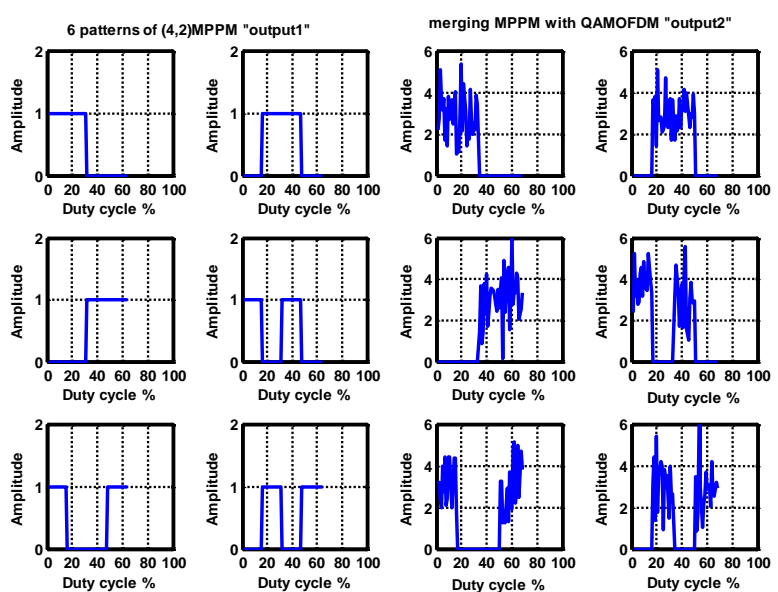
Fig. 3: D) Dividing A PWM Pulse with 50\% Dimming Into $A(W=4, P=$ 2) MPPM Symbol and Bipolar OFDM Signal Transmitted at the "on" Period of $(4,2)$ MPPM Patterns.

Presuming that 4-QAM OFDM symbols are transmitted on the PWM pulses. As describing in Fig. 3 (A), the duty cycle of a PWM pulse is reduced from $100 \%$ to $50 \%$. There is no space for excess signal transmission when the duty cycle is $100 \%$. When the duty cycle of PWM pulses is $100 \%$, symbol rate can be kept constant and there is no need and no space for excess signal transmission. When we dim the LED brightness and reduce the duty cycle to $50 \%$, the required symbol rate should be twice as high as the initial symbol rate to keep constant bit rate which pushes up the receiver sensitivity according to Eq. (8). However, the reduction of duty cycle also produces extra spaces. These spaces can be used for generating various pulse patterns to carry more information which we call pattern effect.

Thus, at Fig. 3 (B) PWM pulses with 50\% dimming are divided into PPM with the same duty cycle then PPM dimming pulse combines with 4-QAM OFDM signal to transmit through the channel.

In Fig. $3(\mathrm{C})$, the 50\% PWM pulse can be divided into two parts which will be rearranged or permutated on the $w=4$ slots of an OPPM symbol with the same duration and duty cycle. After that there are $((w-p+1)$ i.e $(4-2+1=3))$ patterns of each OPPM symbol. The width of optical pulses can be set sufficiently narrow to achieve high transmission efficiency then each pattern of OPPM dimming pulses combines with a 4-QAM OFDM signal.

Finally, in Fig. 3 (D) PWM pulses are divided into multi-pulses and then these pulses rearrange in the different time slots of an MPPM symbol with the same duty cycle. The generated pattern effect can be utilized to transmit excess signal without varying the LED brightness. MPPM symbol can transmit extra $\log _{2} 6$ bits beyond conventional PWM signal where each MPPM symbol with two pulses of four time slots is indicated as a $(4,2)$ MPPM symbol. Variable M-QAM OFDM signal is transmitted on each pattern of MPPM dimming control pulses.

The excess data rate contributed by PPM, a (w, p) OPPM [26], and a (w, p) MPPM symbol can be described as [22- 23]:

$$
\begin{aligned}
& R_{P P M}=\frac{\log _{2}\left(L_{P P M}\right)}{L_{P P M} T_{P W M}} \\
& R_{O P P M}=\frac{\log _{2}\left(L_{O P P M}\right)}{\left(\frac{w}{p}\right) T_{P W M}} \\
& R_{M P P M}=\frac{\log _{2}\left(L_{M P P M}\right)}{T_{P W M}}
\end{aligned}
$$

Where $L_{P P M}$ presents subintervals of PPM, the period of original PWM signal is $\mathrm{T}_{\mathrm{PWM}}$, subintervals of OPPM are $L_{O P P M}=w-p+1$, subintervals of MPPM are $L_{M P P M}=\mathrm{C}_{p}^{w}$, the total number of slots of an OPPM and MPPM symbol is $\mathrm{w}$, and the number of pulses in each OPPM and MPPM symbol is $\mathrm{p}$.

\subsection{Merging schemes model and evaluating parameters}

Now, this work will modify the mathematical background of merging MPPM with M-QAM OFDM [22-24] to provide a generalized mathematical model that describes the performance due to other types of merging (i.e PPM and OPPM with M-QAM DCO OFDM).

When OFDM signal is $v(t)$ and $N(t)_{\alpha}$ is the signals of PPM, OPPM and MPPM, the resultant merging signal according to aforementioned system schematic is $Y(t)_{\varpi}$ which can be written
$Y(t)_{\varpi}=v(t) N(t)_{\alpha}$

Where $\alpha$ represents the PPM, OPPM or MPPM, $\varpi$ is QAM OFDM PPM, QAM OFDM OPPM, or QAM OFDM MPPM.

The noise power in terms of symbol rate is altered and it will reflect the effect of merging. The $\sigma^{2}(\mathrm{~B}, \mathrm{R})$ in Eq. (3) is modified due to merging with PPM, OPPM, and MPPM which will be provided in Eqs. (8), (9), (11) and (12).

A large excess of symbol rate will be caused by the duty cycle reduction. The BER upper bound of a VLC system by considering the BER of M-QAM [27] can be given as

$$
B E R_{Q A M} \leq 0.2 \exp \left[\frac{-1.5\left(R \mathrm{P}_{r} M_{\text {index }}\right)^{2} f(t)^{2}}{\left(M_{Q A M}-1\right) \sigma^{2}\left(B, \mathrm{P}_{r}\right)}\right]
$$

Where $\sigma^{2}(\mathrm{~B}, \mathrm{R})$ in Eq. (3) is replaced by $\sigma^{2}\left(B, \mathrm{P}_{r}\right)$ to represent the BER of M-QAM, recalling that $\mathrm{P}_{r}$ is the received carrier power.

BER less than $10^{-3}$ is usually required to fulfill reliable transmission [28]. Modifying the required receiver sensitivity $P_{\text {sens }}$ to achieve the target BER based on $[22,24]$ is required to get a generalized formula that takes into account PPM and OPPM with the previously addressed MPPM. This is done to provide:

$\mathrm{P}_{\text {sens }_{\varpi}}=\frac{\sigma\left(B, \mathrm{P}_{\text {sen }}\right)}{\Re M_{\text {index }}} \sqrt{\frac{\left(1-M_{Q A M}\right) \ln \left(\frac{10^{-3}}{0.2}\right)}{1.5 f(t)^{2}}}$

The increased symbol rate will push up the required receiver sensitivity when the duty cycle is decreased because of dimming control. The received optical power is fixed for a given light source in a certain channel model. If it is lower than the required sensitivity, keeping reliable transmission becomes impossible. The total number of transmitted bit rate should be adjusted according to the $\mathrm{P}_{\text {sens }}$ in order to satisfy BER less than $10^{-3}$. This means i) fixed rate transmission can be attained at large duty cycle, and ii) data rate should be lowered at smaller on time. To keep the data rate constant, the total bits proportion must be transmitted at smaller duty cycle [22]. For each merging VLC scheme, the turning point $\mathrm{D}_{\mathrm{T}}$ as described in [22] of duty cycle should be calculated independently by solving the modified required LED power (the transmitted peak LED power when applying dimming to achieve BER below $10^{-3}$ ) for each merging scheme that is presented as follows:

$$
\mathrm{P}_{r e q_{\sigma}}=\frac{\sigma\left(\frac{R_{0}}{D_{T}}, \mathrm{P}_{r}\right)}{\mathfrak{R} M_{\text {index }}} \sqrt{\frac{\left(1-M_{Q A M}\right) \ln \left(\frac{10^{-3}}{0.2}\right)}{1.5 f(t)^{2}}}
$$

Constant rate transmission can be realized when duty cycle exceeds $D_{T}$. However, when the duty cycle is less than $D_{T}$, data rate should be reduced accordingly to adapt to required receiver sensitivity. This technique can be further described as [22, 24]

$$
R_{\max }=\frac{R_{0} D}{D_{T}}, 0 \leq D \leq D_{T} \& R_{\max }=R_{0}, D_{T} \leq D \leq 1
$$

BER less than $10^{-3}$ can be achieved within the whole range of duty cycle from 1 tending to 0 if the transmitted symbol rate is always maintained less than $\mathrm{R}_{\max }$. Consequently, this technique can maximize data rate and retain dependable communication quality simultaneously. 
Presuming compensated distortion and perfect synchronization, a bit error occurs when one or more optical pulses are lost and thus the system BER can be presented as

$B E R_{\varpi}=1-P_{\text {correct }}$

$=1-\sum_{l=0}^{g}\left\{(x)\left(e^{-\delta}\right)^{l}\left(1-e^{-\delta}\right)^{g-t}\left[\mu_{\alpha, l}(1-D)+\mu_{|c|, t} D\left(1-B E R_{Q A M}\right)\right]\right\}$

Where $\quad x_{P P M}=L_{P P M}, \quad \mathrm{x}_{O P P M}=L_{O P P M}=w-p+1$,

$x_{M P P M}=L_{M P P M}=\mathrm{C}_{w}^{p}$, the number of photons each optical pulse is $\delta$. The coefficient $\mu_{|c|, t}$ and $\mu_{\alpha, t}$ respectively denote OFDM intercarrier interference $(|c|)$ and the impact during (PPM, OPPM, and MPPM) signal recovery is when $\imath$ pulses are distorted or lost, both from 1 to 0 reduce with the increase of $\imath$. Here we consider a scenario when no (PPM, OPPM, or MPPM) pulse is lost.

This means both $\mu_{k \mid .0}$ and $\mu_{\alpha, 0}$ are equal to 1 . When OFDM symbols are modulated onto PPM, OPPM, and MPPM dimming control pulses, the intensity of DC-bias is usually large enough so that

$$
B E R_{\varpi}=D \times B E R_{Q A M}
$$

The parameters, that are fed to the mathematical background and used to predict the performance of the merged systems in the following section, are presented in table 1. These parameters are extracted from related famous literature to provide the ability to compare the results [20], [22]

The implementation of the mathematical model of the merged system in the following section using MATLAB program is represented in a block diagram shown in Fig. 4 where a metric of the system one calculates the required LED power and/or the required receiver sensitivity to achieve a given bit error rate better that $10^{-3}$ for a given bit rate.

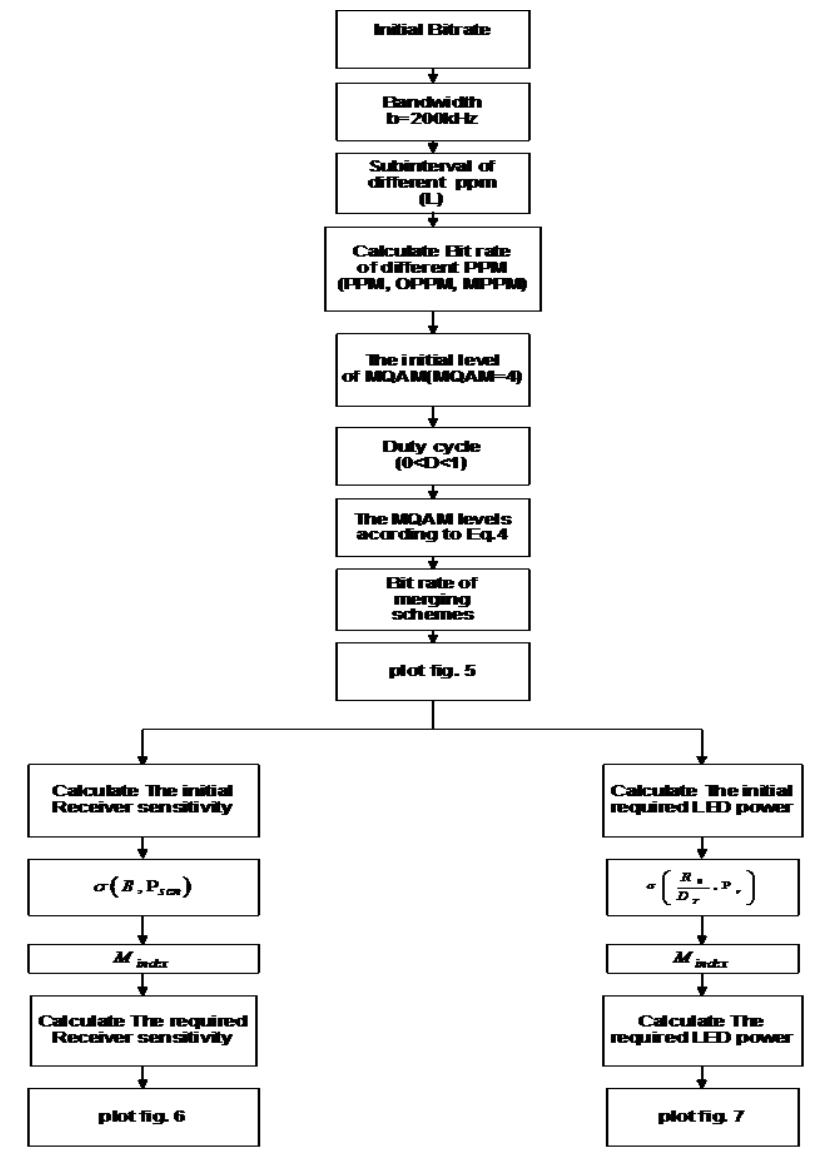

Fig. 4: A Block Diagram Showing the Sequence of the Mathematical Model.

\section{Numerical results and discussion}

In this section, the merging between M-QAM DCO OFDM signal with PWM, PPM, OPPM, and MPPM light pulses is simulated and evaluated. The first property to be calculated is the bit rate variation with the duty ratio for the different modulation and dimming techniques assuming an initial rate of $50 \mathrm{Mb} / \mathrm{s}$ without dimming. This is necessary because one could not keep the input data rate constant for all the proposed modulation techniques. So that there is a relation between the number of bits per symbol and the states $\mathrm{M}$ of the symbol such as $\mathrm{n}=$ number of bits in a symbol= $\log _{2} \mathrm{M}$. Both $\mathrm{M}$ and $\mathrm{n}$ must be integers.

Table 1: Parameters of Indoor VLC Communication Transceiver

\begin{tabular}{llll}
\hline Parameters & Values & Parameters & Values \\
\hline $\begin{array}{l}\text { absolute tempera- } \\
\text { ture } \mathrm{T}_{\mathrm{k}}\end{array}$ & $295 \mathrm{~K}$ & $\begin{array}{l}\text { Noise bandwidth factor } \\
\left(\mathrm{I}_{2}\right)\end{array}$ & 0.562 \\
$\begin{array}{l}\text { Background } \\
\text { current }\left(\mathrm{I}_{\mathrm{bg}}\right)\end{array}$ & $5100 \mathrm{uA}$ & $\begin{array}{l}\text { Open loop voltage gain } \\
(\mathrm{G})\end{array}$ & 10 \\
$\begin{array}{l}\text { Open loop volt- } \\
\text { age gain }(\mathrm{G})\end{array}$ & 10 & $\begin{array}{l}\text { Physical area of photo- } \\
\text { detector }(\mathrm{A})\end{array}$ & $10^{-4} \mathrm{~m}^{2}$ \\
$\begin{array}{l}\text { FET channel } \\
\text { noise factor }(\Gamma)\end{array}$ & 1.5 & $\begin{array}{l}\text { Receiver's field of } \\
\text { view }(\mathrm{FOV})\end{array}$ & $170 \mathrm{deg}$ \\
$\begin{array}{l}\text { FET transcon- } \\
\text { ductance }\left(\mathrm{g}_{\mathrm{m}}\right)\end{array}$ & $30 \mathrm{mS}$ & $\begin{array}{l}\text { Room size } \\
\text { length*width*height) }\end{array}$ & $5 \mathrm{~m} * 5 \mathrm{~m} * 3 \mathrm{~m}$ \\
$\begin{array}{l}\text { Fixed capacitance } \\
(\mathrm{M})\end{array}$ & $112 \mathrm{pF} / \mathrm{cm}^{2}$ & $\begin{array}{l}\text { Responsivity of photo } \\
\text { detector }(\Re)\end{array}$ & $1 \mathrm{~A} / \mathrm{W}$ \\
$\quad \mathrm{I}_{3}$ & 0.0868 & $\begin{array}{l}\text { Transmitter's semi- } \\
\text { angle } \\
\text { Transmitter's modula- } \\
\text { tion index }\left(\mathrm{M}_{\text {index }}\right)\end{array}$ & $60 \mathrm{deg}$ \\
$\begin{array}{l}\text { Location of LED } \\
\text { lamp }\end{array}$ & $2.5 \mathrm{~m}$, \\
\hline
\end{tabular}

Evaluation is done by utilizing blue filter at the receiver that can extend the modulation bandwidth to approximately $20 \mathrm{MHz}$ [5]. The initial data rate is assumed to be $50 \mathrm{Mb} / \mathrm{s}$ [20]. The frequency of PWM and MPPM pulses is set at $200 \mathrm{kHz}$ to avoid flickering [22]. The same frequency is adjusted for the proposed schemes (i.e PPM, OPPM). The variable M-QAM levels with all merging scenarios under each duty cycle are given in Table 2 by applying Eqs. (4) and (10) [22- 23]. The results are carried target less than $10^{-3}$ BER performance.

Table 2: Adaptive M-QAM Levels for All Merged Scenarios

\begin{tabular}{llllllll} 
Duty cycle (D) & 1.0 & $0.9-0.7$ & 0.6 & 0.5 & 0.4 & 0.3 & 0.2 \\
\hline M-QAM levels (M) & 4 & 8 & 16 & 16 & 32 & 128 & 1024
\end{tabular}

Figure 5 represents the relation between bit rate and duty cycle for schemes combining (PWM, PPM, OPPM, and MPPM) light pulses and M-QAM OFDM signal. It is shown that decreasing the designed duty cycle (represent dimming ratio) enhances the operating bit rate for all merging scenarios. At specific duty cycle, the optimum performance is observed with OFDM-MPPM while the lowest performance is associated with OFDM-PWM. This does not mean that OFDM-MPPM is the optimum choice for this work. Other performance characteristics should be taken into consideration and will be presented in Fig. 6, 7 . 


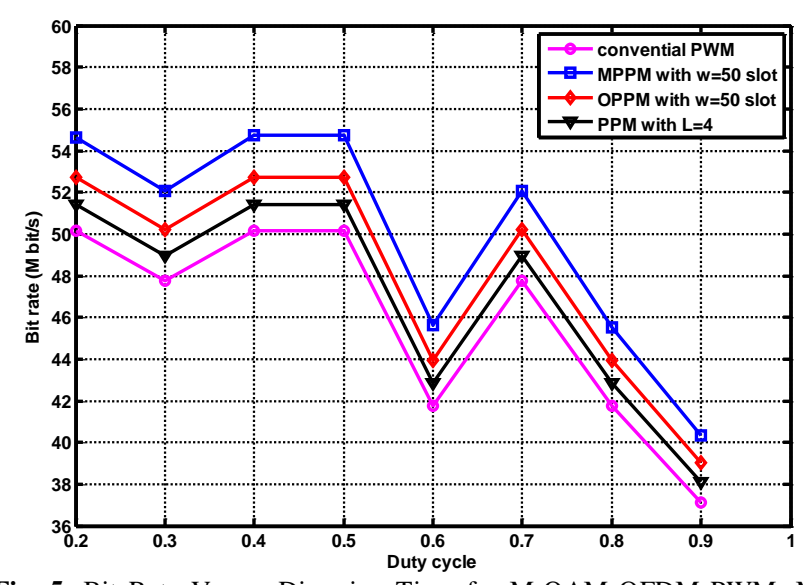

Fig. 5: Bit Rate Versus Dimming Time for M-QAM OFDM PWM, MQAM OFDM MPPM, M-QAM OFDM OPPM, and M-QAM OFDM PPM Merging Techniques Respectively (Where $\mathrm{R}_{0}=50 \mathrm{Mbit} / \mathrm{Sec}, \mathrm{B}=200 \mathrm{khz}$, $\mathrm{L}=4$ Subinterval and $\mathrm{W}=50$ Slots)

While increasing the designed duty cycle for all the merging schemes, the required receiver sensitivity relaxes as shown in Fig. 6 . This agreement with the logical point of view is presented in Fig. 5 where increasing duty cycle, the bit rate lowers and the required receiver sensitivity relaxes. It is noteworthy to mention that the higher the required receiver sensitivity, the more complex and cost for receiver circuit design.

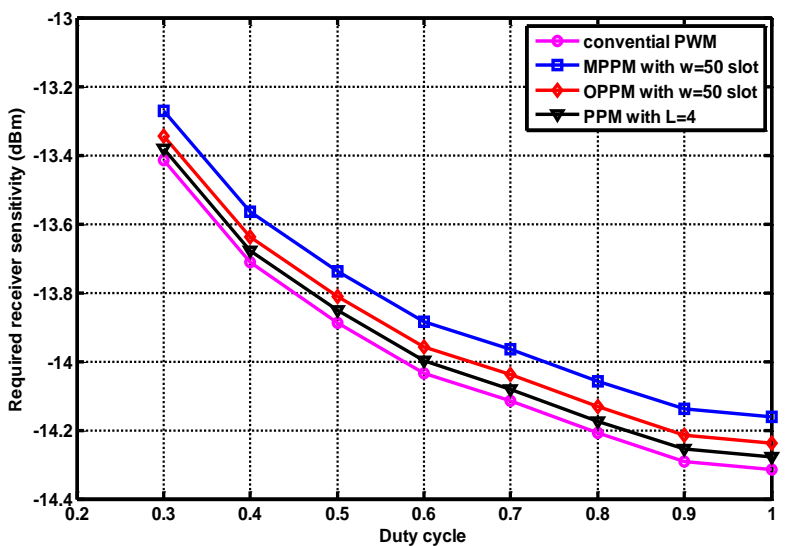

Fig. 6: Required Receiver Sensitivity Versus with Duty Cycle for M-QAM OFDM PWM, M-QAM OFDM MPPM, M-QAM OFDM OPPM, And MQAM OFDM PPM Merging Techniques Respectively (Where $\mathrm{M}_{\text {Index }}=$ $0.2, \mathrm{R}_{0}=50 \mathrm{Mbit} / \mathrm{Sec}, \mathrm{B}=200 \mathrm{khz}, \mathrm{L}=4$ Subinterval and $\mathrm{W}=50$ Slots).

Figure 7 represents the relation between required LED power and duty cycle to satisfy BER under $10^{-3}$ for schemes combining (PWM, PPM, OPPM, and MPPM) light pulses with M-QAM OFDM signal. At lower duty cycle (i.e high bit rate and required receiver sensitivity), the required LED power is higher for all merging techniques. The M-QAM OFDM signal merged with PPM light pulses requires the lowest LED power compared to other merging techniques. This is a result of the superior powersaving phenomena that is associated with PPM scheme and proved (without merging) in many literatures [1], [18- 19], [26]. At higher duty cycle, the difference between required LED power in all merging techniques becomes negligible.

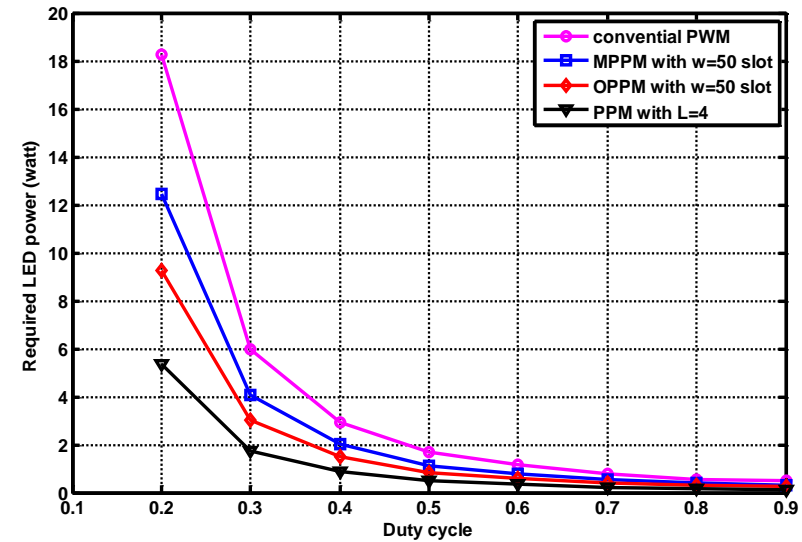

Fig. 7: Required LED Power Versus Duty Cycle for M-QAM OFDM PWM, M-QAM OFDM MPPM, M-QAM OFDM OPPM, and M-QAM OFDM PPM Merging Techniques Respectively (Where $\mathrm{M}_{\text {Index }}=0.2$, $\mathrm{R}_{0}=50 \mathrm{Mbit} / \mathrm{Sec}, \mathrm{B}=200 \mathrm{khz}, \mathrm{L}=4$ Subinterval and $\mathrm{W}=50$ Slots).

Figure 8 depicts the relation between the logarithmic scale of bit error rate (BER) and signal-to-noise ratio (SNR) for merging (PWM, PPM, OPPM, and MPPM) light pulses with M-QAM DCO OFDM signal at M-QAM level=16, 50Mbit/sec, and different dimming level (i. e Fig. 8 (A) with $20 \%$ dimming, Fig. 8 (B) with 50\% dimming, Fig. 8 (C) with70\% dimming).

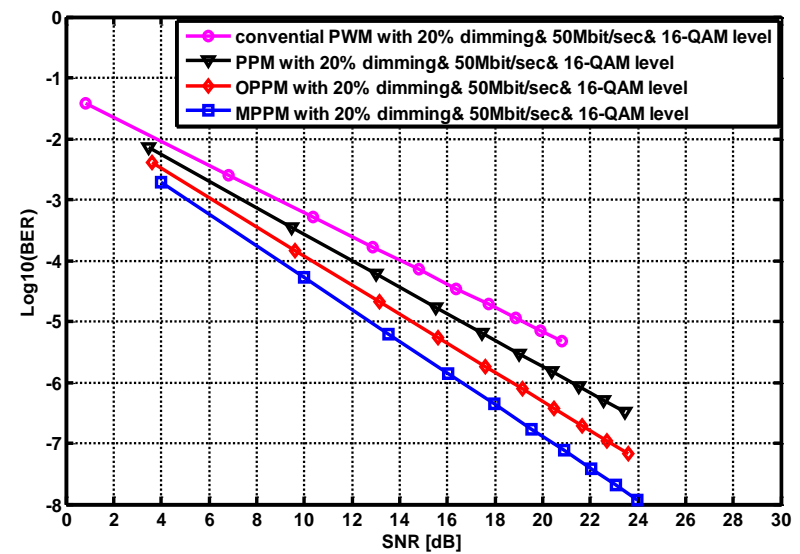

Fig. 8: A) Bit Error Rate (BER) Versus with Signal-To-Noise Ratio (SNR) for M-QAM OFDM PWM, M-QAM OFDM MPPM, M-QAM OFDM OPPM, and M-QAM OFDM PPM Merging Techniques Respectively with $20 \%$ Dimming.

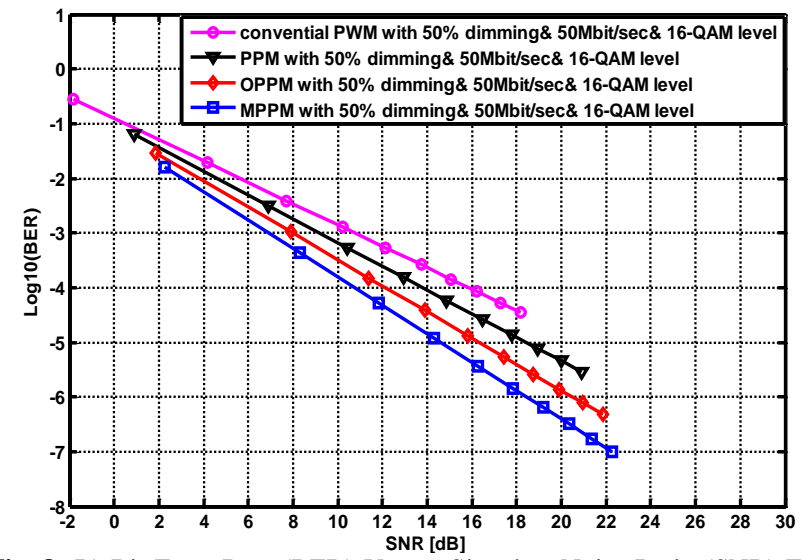

Fig. 8: B) Bit Error Rate (BER) Versus Signal-to-Noise Ratio (SNR) For M-QAM OFDM PWM, M-QAM OFDM MPPM, M-QAM OFDM OPPM, and M-QAM OFDM PPM Merging Techniques Respectively with 50\% Dimming. 


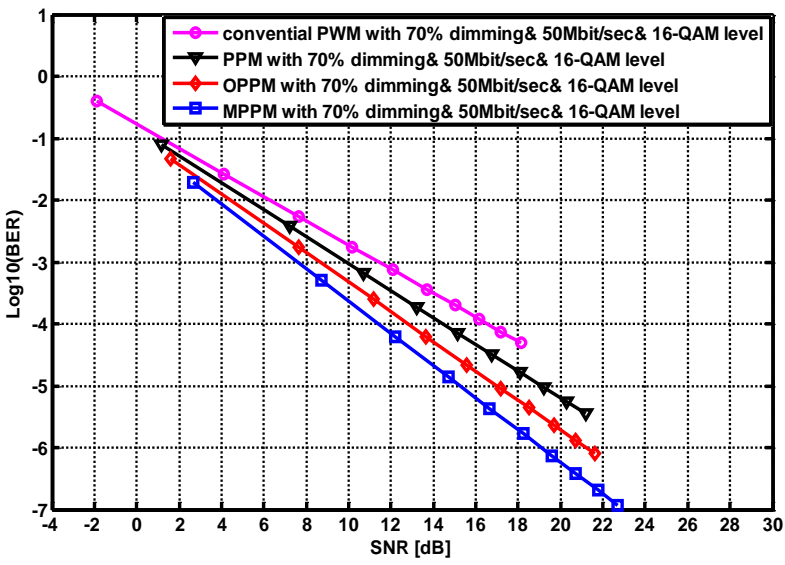

Fig. 8: C) Bit Error Rate (BER) Versus With Signal-to-Noise Ratio (SNR) for M-QAM OFDM PWM, M-QAM OFDM MPPM, M-QAM OFDM OPPM, and M-QAM OFDM PPM Merging Techniques Respectively with $70 \%$ Dimming.

Figure 9 represents the relation between the required receiver sensitivity and the logarithmic scale of BER for all merging schemes while using the same parameters of Fig. 8.

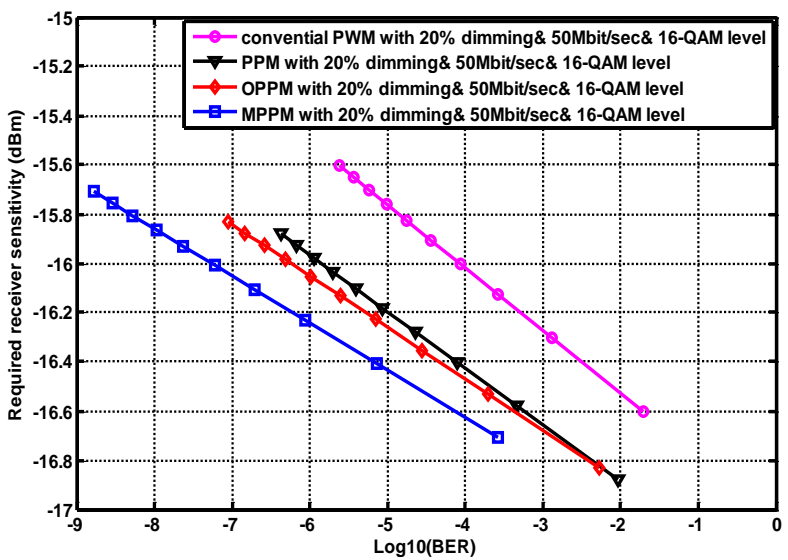

Fig. 9: A) The Required Receiver Sensitivity Versus with Bit Error Rate (BER) for M-QAM OFDM PWM, M-QAM OFDM MPPM, M-QAM OFDM OPPM, and M-QAM OFDM PPM Merging Techniques Respectively with $20 \%$ Dimming.

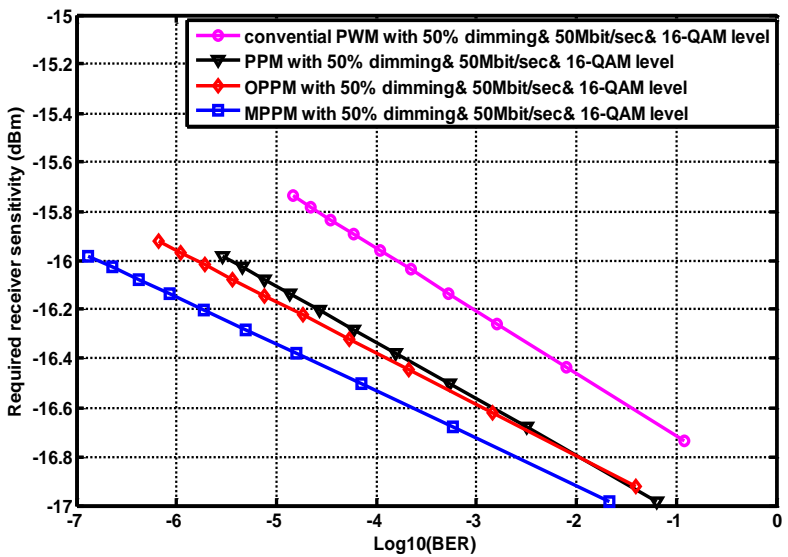

Fig. 9: B) The Required Receiver Sensitivity Versus with Bit Error Rate (BER) for M-QAM OFDM PWM, M-QAM OFDM MPPM, M-QAM OFDM OPPM, and M-QAM OFDM PPM Merging Techniques Respectively with $50 \%$ Dimming.

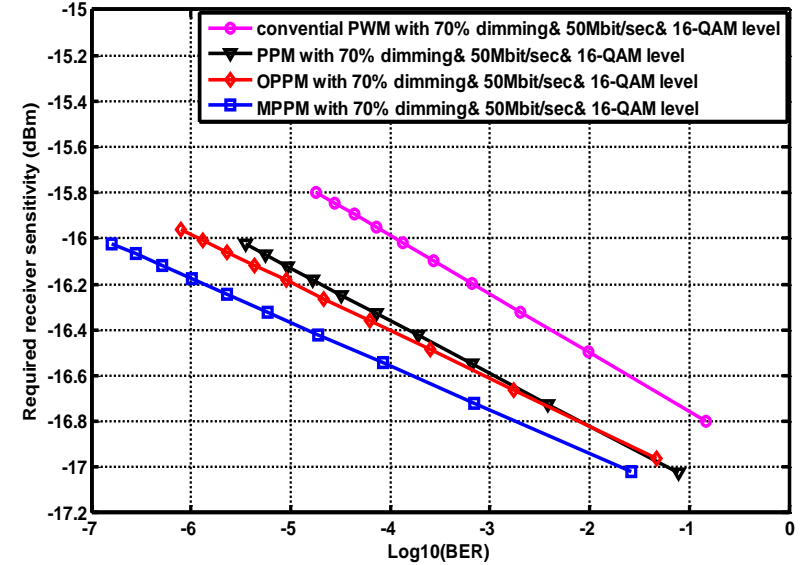

Fig. 9: C) The Required Receiver Sensitivity Versus with Bit Error Rate (BER) for M-QAM OFDM PWM, M-QAM OFDM MPPM, M-QAM OFDM OPPM, and M-QAM OFDM PPM Merging Techniques Respectively with $70 \%$ Dimming.

Reviewing the results, the introduced targets are 1) harmony with SCM standard transmission for PPM [1, 26], and 2) a medium level of scheme complexity that is increased with increasing (w). The results for performance investigation and evaluation for the merging schemes are carried with $\mathrm{L}=4$ (i.e. $\mathrm{PPM})$ and $(\mathrm{w}=50)$ for MPPM and OPPM.

\section{Conclusion}

In this work, an exploring on VLC MCM schemes is carried out. These schemes consist of merging between M-QAM DCO OFDM signals with PWM, PPM, OPPM or MPPM dimming control pulses. The mathematical formulation for merging M-QAM DCO OFDM with PPM and OPPM is worked out and the resulting signal is evaluated compared to the conventional merging (i.e MQAM DCO OFDM with PWM) under BER less than $10^{-3}$. In VLC MCM schemes, the high bit rate operation conditions are essentially targeted with the expected price that will be paid at scheme complexity. Receiver sensitivity and the required LED power are satisfied for dimming control mechanism in indoor VLC. Conventional merging suffers from the highest required LED power and receiver sensitivity while it achieves the lowest bit rate. For high bit rate performance, it is observed that merging with MPPM achieves the highest possible bit rate. With the number of slots $(\mathrm{w}=50)$ and duty cycle $(\mathrm{D}=0.3)$, the achieved bit rate is 52.08069 $\mathrm{Mbit} / \mathrm{s}$ while the required LED power is 4.0788 watt, the receiver sensitivity is $-13.2728 \mathrm{dBm}$. On the other hand, it requires more power and the highest receiver sensitivity but still lower than those observed for the conventional merging in the required LED power. PPM merged with M-QAM DCO OFDM achieves the optimum power saving performance and the lowest required receiver sensitivity among the presented merging schemes, but with the lowest bit rate performance excluding the conventional merging. With the number of subintervals $(L=4)$ and at $(D=0.3)$, the required LED power hits a lower level of 1.7585 watt, a receiver sensitivity of $13.3818 \mathrm{dBm}$, and the bit rate of $48.9690 \mathrm{Mbit} / \mathrm{s}$.

\section{References}

[1] F. Zafar, Dil Karunatilaka, And Raj Parthiban, "Dimming Schemes for Visible Light Communication: The State of Research," IEEE Wireless Commun., vol. 22, no. 2, pp. 29-35, July, 2015. Available online: https://ieeexplore.ieee.org/document/7096282/.

[2] Min Wang, Jun Wu, Wei Yu, Hanli Wang, Jiehui Li, Jianyang Shi, and Chong Luo, "Efficient Coding Modulation and Seamless Rate Adaptation for Visible Light Communications," IEEE Wireless Commun. Mag., vol. 22, no. 2, pp. 86-93, April, 2015. Available online: https://ieeexplore.ieee.org/document/7096290/.

[3] T. Komine and M. Nakagawa, "Fundamental Analysis for VisibleLight Communication System Using LED Lights," IEEE Trans. 
Consum. Electron., vol. 50, no. 1, pp. 100-107, 2004. Available online: https://ieeexplore.ieee.org/document/1277847/.

[4] M. Anand and N. Kumar, "New, Effective and Efficient Dimming and Modulation Technique for Visible Light Communication," 2014 IEEE 79Th Veh. Technol. Conf., pp. 1-4, 2014. Available online: https://ieeexplore.ieee.org/document/7023004/.

[5] H. Le Minh et al., "100-Mb/s NRZ Visible Light Communication Using a Postequalized White LED," IEEE Photonics Technol. Lett. vol. 21, no. 15, pp. 1063-1065, 2009. Available online: https://ieeexplore.ieee.org/document/4926203/.

[6] H. Li, X. Chen, B. Huang, D. Tang, and H. Chen, "High Bandwidth Visible Light Communications Based on a Postequalization Circuit," IEEE Photonics Technol. Lett., vol. 26, no. 2, pp. 119 122, 2014. Available online: https://ieeexplore.ieee.org/document/6657715/.

[7] R. D. Roberts, "IEEE 802 . 15 . 7 Visible Light Communication : Modulation Schemes and Dimming Support," IEEE Commun. Mag., vol. 50, no. 3, pp. 72-82, 2012. Available online: http://jglobal.jst.go.jp/en/public/20090422/201202205419842751.

[8] Y. Tanaka, S. Haruyama, and M. Nakagawa, "Wireless Optical Transmissions with White Colored LED for Wireless Home Links,' 11th IEEE Int. Symp. Pers. Indoor Mob. Radio Commun. PIMRC 2000. Proc. (Cat. No.00TH8525), vol. 2, pp. 1325-1329, 2000. Available online: https://ieeexplore.ieee.org/document/881634/.

[9] S. H. Lee, S.-Y. Jung, and J. K. Kwon, "Modulation and coding for dimmable visible light communication," IEEE Commun. Mag., vol. 53, no. 2, pp. 136-143, 2015. Available online: https://ieeexplore.iee.org/document/7045402.

[10] I. Din and H. Kim, "Energy-Efficient Brightness Control and Data Transmission for Visible Light Communication," IEEE Photonics Technol. Lett.,vol. 26, no. 8, pp. 781-784, 2014. Available online: https://ieeexplore.ieee.org/document/6740016/.

[11] R. Mesleh, H. Elgala, and H. Haas, "On the Performance of Different OFDM Based Optical Wireless Communication Systems," J. Opt. Commun. Netw, vol. 3, no. 8, pp. 620-628, 2011. Available online: https://ieeexplore.ieee.org/document/5967993/.

[12] Mohammed Abd Elkarim, Nazmi A. Mohammed and Moustafa H. Aly, "Exploring the performance of indoor localization systems based on VLC-RSSI including the effect of NLOS components using two LED lighting systems," Optical Engineering, vol. 54, no. 10, pp. 105-110 (1-9), Oct. 2015. Available online: https://doi.org/10.1117/1.OE.54.10.105110

[13] N. a Mohammed and M. A. Elkarim, "Exploring the effect of diffuse reflection on indoor localization systems based on RSSIVLC," Optics Express, vol. 23, no. 16, pp. 20297-20313, 2015 Available online: https://doi.org/10.1364/OE.23.020297.

[14] M. Mehrabi, S. Lafond, and L. Wang, "Frame Synchronization of Live Video Streams Using Visible Light Communication," 2015 IEEE Int. Symp. Multimed. , pp. 128-131, 2015. Available online: https://ieeexplore.ieee.org/document/7442311/.

[15] T. Yamazato et al., "The Uplink Visible Light Communication Beacon System for Universal Traffic Management," IEEE Access, vol. 5, no. X, pp. 22282-22290, 2017. Available online: https://ieeexplore.ieee.org/document/7442311/.

[16] K. Nakamura, I. Mizukoshi, and M. Hanawa, "Optical wireless transmission of $405 \mathrm{~nm}, 145 \mathrm{Gbit} / \mathrm{s}$ optical IM/DD-OFDM signals through a 48 m underwater channel," Optics Express, vol. 23, no. 2, pp. 1558-1566, 2015. Available online: https://doi.org/10.1364/OE.23.001558.

[17] D. R. Dhatchayeny, A. Sewaiwar, S. V Tiwari, and Y. H. Chung, "Experimental Biomedical EEG Signal Transmission Using VLC," IEEE Sens. J., vol. 15, no. 10, pp. 5386-5387, 2015. Available online: https://ieeexplore.ieee.org/document/7151783/.

[18] A. Jovicic, J. Li, and T. Richardson, "Visible light communication: Opportunities, challenges and the path to market," IEEE Commun Mag., vol. 51, no. 12, pp. 26-32, 2013. Available online: https://ieeexplore.ieee.org/document/6685754/.

[19] Mohamed Sufyan, Islim, Harald Haas, "Modulation Techniques for Li - Fi," Geol. Bull. China, vol. 14, no. 1-2, pp. 1-12, 2016 Available

online: http://www.cnki.net/kcms/detail/34.1294.TN.20160413.1658.002.h $\mathrm{ml}$.

[20] Z. Wang, W.-D. Zhong, C. Yu, J. Chen, C. P. S. Francois, and W Chen, "Performance of Dimming Control Scheme in Visible Light Communication System," Optics Express, vol. 20, no. 17, pp. 18861-18868, 2012. Available online: https://doi.org/10.1364/OE.20.018861.

[21] J. Armstrong, "OFDM for Optical Communications. Elsevier, Amsterdam,” J. Lightwave Technol., vol. 27, no. 3, pp. 189-204,
2009

Available

online: https://ieeexplore.ieee.org/document/4785281/.

[22] X. You, J. Chen, H. Zheng, and C. Yu, "Efficient Data Transmission Using MPPM Dimming Control in Indoor Visible Light Communication," IEEE Photonics J., vol. 7, no. 4, 2015 Available online: https://ieeexplore.ieee.org/document/7145378/.

[23] J. Chen, X. You, H. Zheng, and C. Yu, "Excess Signal transmission with Dimming Control Pattern in Indoor Visible Light Communication Systems," Optoelectron. Devices Integr. V, vol. 9270, no. 14, pp. 1-8, 2014. Available online: https://doi.org/10.1117/12.2073504.

[24] K. Forest and G. Question, "MPPM Dimming Control for OFDMBased Visible Light Communication", 2014 IEEE international Conf., vol. 15, no. D, pp. 268-272, 2015. Available online: https://ieeexplore.ieee.org/document/7024807/.

[25] H. Elgala and T. D. C. Little, "Reverse Polarity Optical-OFDM (RPO-OFDM): Dimming Compatible OFDM for Gigabit VLC Links," Optics Express, vol. 21, no. 20, pp. 24288 -24299, 2013. Available online: https://www.ncbi.nlm.nih.gov/pubmed/24104338.

[26] Nazmy Azzam, Moustafa H. Aly and A.K. AboulSeoud, "Bandwidth and Power Efficiency of Various PPM Schemes for Indoor Wireless Optical Communications," Proceeding of the $26^{\text {th }}$ National Radio Science Conference, NRSC'2009, Cairo, Egypt, pp. C10 (1-10), Mar. 18-20, 2009. Available online: https://ieeexplore.ieee.org/document/5233992/.

[27] A. J. Goldsmith, S. Chua, and A. Member, "Variable-rate variablepower MQAM for Fading Channels," , IEEE transactions on comm. vol. 45, no. 10, pp. 1218-1230, 1997. Available online: https://ieeexplore.iee.org/document/634685/.

[28] R.-J. Essiambre, G. Kramer, P. J. Winzer, G. J. Foschini, and B. Goebel, "Capacity Limits of Optical Fiber Networks," J. Lightwave Technol., vol. 28, no. 4, pp. 662-701, 2010. Available online: https://ieeexplore.ieee.org/document/5420239/. 